EPJ Web of Conferences 41, 02008 (2013)

DOI: $10.1051 /$ epjconf/20134102008

(C) Owned by the authors, published by EDP Sciences, 2013

\title{
Attosecond intramolecular electron dynamics
}

\author{
A. Becker ${ }^{1, a}$, N. Takemoto ${ }^{1,2}$, A. Picón ${ }^{1, b}$, and A. Jaroń-Becker ${ }^{1}$ \\ 1 JILA and Department of Physics, University of Colorado, Boulder, CO 80309, USA \\ 2 Department of Chemical Physics, Weizmann Institute of Science, 76100 Rehovot, Israel
}

\begin{abstract}
We present results of numerical simulations indicating a complex laser driven electron dynamics inside simple molecular systems on the attosecond time scale. This attosecond electron dynamics influences the instant of ionization of the molecule and the final electron momentum distributions.
\end{abstract}

\section{Introduction}

The emission of an electron from an atom or molecule is presumably one of the simplest but most central processes in physics and chemistry. It is the doorway step to many other strong-field processes, such as high harmonic and attosecond pulse generation, molecular dissociation, ultrafast molecular imaging, electron diffraction, angular streaking, etc. Widely used ionization pictures are based on the quasistatic approximation for the laser field. In tunnel ionization, the combined potential of the Coulomb attraction of the atomic or molecular core and the laser electric field form a barrier through which the electron can escape. Some molecules, such as the hydrogen molecular ion, show more complex ionization mechanisms. Because of a strong coupling between two quasidegenerate states (so-called charge-resonant states) in $\mathrm{H}_{2}^{+}$at intermediate internuclear distances, the energy level at one of the protons is lifted, while it is decreased at the other by the laser electric field. At high field strengths, the upper state lies above the internal Coulomb barrier and the electron is ionized efficiently (called enhanced ionization $[1,2]$ ). All these quasistatic ionization pictures have in common that the ionization probability is expected to be largest whenever the external field is strongest.

\section{Sub-cycle ionization dynamics}

Our recent results of numerical solutions of the time-dependent Schrödinger equation capturing the interaction of the hydrogen molecular ion with an intense linearly polarized laser pulse however do not agree with the expectation of the quasistatic picture [3]. As shown in Figure 1(b), over one half cycle of the oscillation of the laser electric field the ionization rate of the hydrogen molecular ion can have two maxima but a minimum near the peak of the field. This unexpected multiple ionization burst phenomenon in case of the hydrogen molecular ion becomes specifically apparent when compared to results of a quasistatic ionization theory for the hydrogen atom, which exhibit the expected maxima in ionization at every maximum of the field (Figure 1(a)). Indeed, further theoretical analysis showed that even more than two ionization bursts can occur within a half cycle of the oscillation of the field depending on the parameters of the linearly polarized field [5].

A related unexpected ionization dynamics was observed for the interaction of the hydrogen molecular ion with a circularly polarized laser pulse. The experimentally observed photoelectron momentum distribution was tilted in the same direction as the rotation of the laser electric field and peaked at lower

a e-mail: andreas.becker@colorado.edu

b Present address: Argonne National Laboratory, Argonne, Illinois 60439, USA

This is an Open Access article distributed under the terms of the Creative Commons Attribution License 2.0, which permits unrestricted use, distribution, and reproduction in any medium, provided the original work is properly cited. 

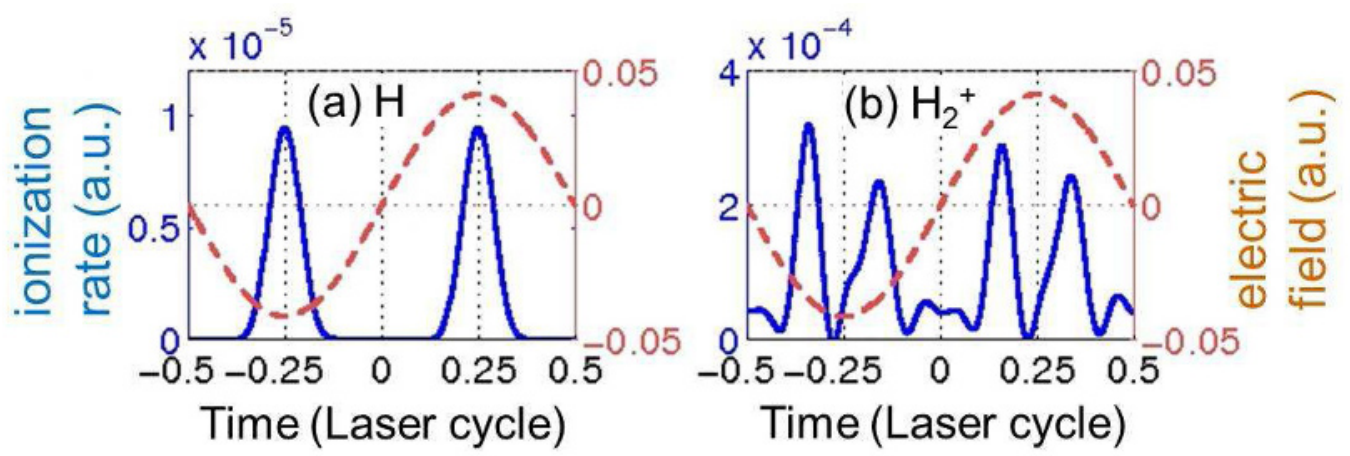

Fig. 1. Ionization rates (solid blue curves) of (a) the hydrogen atom and (b) the hydrogen molecular ion as a function of time over one cycle of a laser pulse [3]. The molecular ionization rates, as obtained by numerical solution of the corresponding time-dependent Schrödinger equation, are accumulated over internuclear separations between 6.75 a.u. and 7.25 a.u. The electric field of the laser pulse is represented by the dashed red curves and the field parameters were wavelength: $800 \mathrm{~nm}$, peak intensity: $6 \times 10^{13} \mathrm{~W} / \mathrm{cm}^{2}$ and pulse duration: $26.69 \mathrm{fs}$.

momenta than expected [4]. Results of our ab-initio numerical simulations reproduced the tilted peak as the main contribution in the calculated electron momentum distribution. The theoretical analysis revealed that the electron wave packet was released 356 as after the laser electric field was aligned along the internuclear axis and with an initial velocity having a component opposite to the acceleration by the field [4].

\section{Transient electron localization on the attosecond time scale}

The unexpected ionization dynamics in the hydrogen molecular ion is closely related to a transient electron localization at one of the protons on the attosecond time scale [6]. The intramolecular electron transfer between the protons is gated at specific electron momenta [7], which results in an intensitydependent electron localization in the dissociating hydrogen molecular ion [6] and the multiple ionization bursts $[3,5,8]$.

We gained further complementary insight into the multiple ionization burst phenomenon by analyzing the electron dynamics in a model consisting of the two charge resonant states $\left(1 s \sigma_{g}\right.$ and $2 p \sigma_{u}$ in the case of hydrogen molecular ion), which is valid when these two states are coupled much more strongly with each other than with the other states in the molecule [5]. Analyzing the general solution of the time-dependent Schrödinger equation of the model in terms of the two Floquet states expressed in form of a series expansion, the time instants $t_{l o c}$ for localization of the electron distribution at one of the protons is given by [3,5] (Hartree atomic units are used):

$$
A\left(t_{l o c}\right)=\frac{m \pi+\chi}{2 d_{g u}} \quad \text { with } \quad m=0, \pm 1, \pm 2, \ldots
$$

where $A$ is the time-dependent vector potential of the laser field, $\chi$ is the mixing angle of the two Floquet states and $d_{g u}$ the dipole transition matrix element. For large $d_{g u}$ and large $A$, the condition can be fulfilled for more than one value of $t_{l o c}$ in a half cycle of the oscillation of the electric field which results in a bunching of the electron localization and the corresponding multiple ionization bursts.

Although the theoretical analysis has been performed and Eq. (1) has been confirmed for the specific case of the hydrogen molecular ion [5], we expect that the general features of the electron dynamics found for $\mathrm{H}_{2}^{+}$hold for other molecules with pair(s) of quasi-degenerate charge resonant states as well. Indeed, results of numerical simulations for the electron dynamics in a linear $\mathrm{H}_{3}^{2+}$ model system, in which the electron dynamics is restricted along the molecular axis, are in qualitative agreement with these expectations. In our model the protons are fixed at equidistant internuclear separations of 5.5 a.u. 

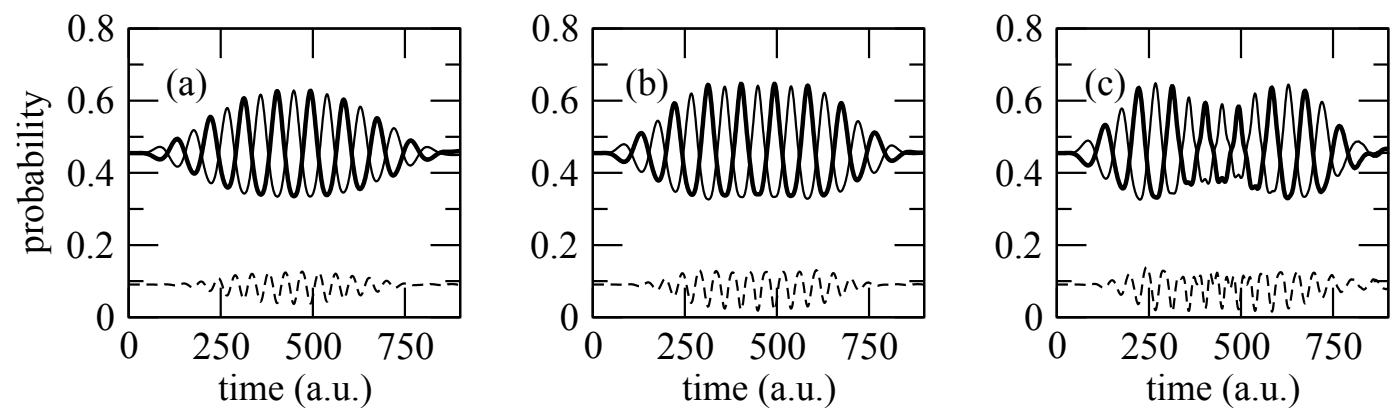

Fig. 2. Results of numerical simulations for the probabilities of electron localization at one of the three protons inside a linear model for $\mathrm{H}_{3}^{2+}$ as a function of time during the interaction with an intense laser pulse. The thick and thin solid lines represent electron localization at the outer two protons, while the dashed line represents the localization at the central proton. The wavelength of the laser field was chosen to be $650 \mathrm{~nm}$ with peak intensities of (a) $5 \times 10^{12} \mathrm{~W} / \mathrm{cm}^{2}$, (b) $1 \times 10^{13} \mathrm{~W} / \mathrm{cm}^{2}$, and (c) $2 \times 10^{13} \mathrm{~W} / \mathrm{cm}^{2}$ for a $\sin ^{2}$-envelope of the pulse.

At this configuration there exists a pair of quasidegenerate charge resonant states, in which the electron is predominantly localized at the outer two of the three protons. The numerical results in Figure 2 show that at low intensities (small vector potential $A$ ) the electron distribution is driven adiabatically between the two protons. At higher intensities we observe a change to a nonadiabatic dynamics near the center of the laser pulse (i.e., for large values of the vector potential $A$ ) in qualitative agreement with the predictions based on Eq. (1). Interestingly, we also observe a decrease in the amplitude of the oscillation of the electron distribution at higher intensities as well.

\section{Acknowledgments}

This work was supported by the U.S. Department of Energy (Chemical Sciences, Geosciences, and Biosciences Division, Office of Basic Energy Sciences).

\section{References}

1. T. Zuo and A.D. Bandrauk, Phys. Rev. A 52, (1995) R2511

2. T. Seideman, M.Yu. Ivanov and P.B. Corkum, Phys. Rev. Lett. 75, (1995) 2819

3. N. Takemoto and A. Becker, Phys. Rev. Lett. 105, (2010) 203004

4. M. Odenweller, N, Takemoto, A. Vredenborg, K. Cole, K. Pahl, J. Titze, L.Ph.H. Schmidt, T. Jahnke, R. Dörner and A. Becker, Phys. Rev. Lett. 107, (2011) 143004

5. N. Takemoto and A. Becker, Phys. Rev. A 84, (2011) 023401

6. I. Kawata, H. Kono and Y. Fujimura, J. Chem. Phys. 110, (1999) 11152

7. F. He, A. Becker and U. Thumm, Phys. Rev. Lett. 101, (2008) 213002

8. N. Takemoto and A. Becker, J. Chem. Phys. 134, (2011) 074309 